International Journal of Engineering \& Technology, 7 (3.27) (2018) 70-72
International Journal of Engineering \& Technology
SPC
Website: www.sciencepubco.com/index.php/IJET
Research paper

\title{
Interactive Natural Image Segmentation and Foreground Extraction
}

\author{
Y. David Solomon Raju ${ }^{1 *}$, D. Krishna Reddy ${ }^{2}$ \\ ${ }^{1}$ Research Scholar, Department of Electronics and Communication Engineering, Rayalaseema University, Kurnool. \\ ${ }^{2}$ Professor, Department of Electronics and Communication Engineering, Chaitanya Bharathi Institute of Technology, Hyderabad, \\ Telangana State, India.
}

\begin{abstract}
Interactive image segmentation is very practical and important problem in computer vision. In this paper a regressive based Green's function is employed to formulate the problem of segmentation. The method is incorporated with different clustering approaches intended to extract the foreground regions from the natural images. The method performance is improved with proper labeling of foreground and background regions, and with more number of cluster regions. The method is evaluated with two standard benchmark datasets and found that the experimental results were promising.
\end{abstract}

Keywords: Segmentation, foreground extraction, clustering, green's polynomial function.

\section{Introduction}

Interactive and semantic image segmentation approaches have attained greater interest in the field of computer vision and deep learning. The work finds its own application in real time world where large labeling is not possible and needs heavy labor [1]. During the past decade many interactive based image segmentation algorithms were proposed such as level set [2], Snakes [3], Watershed [4], Random walkers [5], Graph cuts [6] and many others. In spite of many segmentation models the problem of segmentation for natural images is still in demand.

Interactive segmentation involves a minimal user interaction intended to extract user defined regions and the results obtained with this approach are more accurate than those of automatic image segmentation. This interactive segmentation involves two major requirements (i) It should be able to extract the users required region of interest (ii) It also to be efficient in achieving the considerable feedback. User intuitiveness is carried till the desirable results are achieved. In this method the region of interest are labeled with mouse dragging and it doesn't not require any boundary markings as in the case of [2] [3].

The problem of this labeling can be also termed as the problem of classification, and this interactive-ness can be termed as semi supervised classification. Some of the algorithms like label propagation with local and global consistence [7] and [5] may not be able to produce satisfactory results for natural images.

In this paper, interactive based image segmentation (label classification) is proposed and formulated as a Green's polynomial function.

A clustering approach is employed to partition the image different labeled region which is further refined by a regression based linear polynomial expression. The paper is organized as, section 1 presents the need and the necessity of the natural image segmentation.

\section{Related Work}

In general the label propagation proposed by Wang in [8] formulated the problem as a optimization solution represented as

$\min \left(f^{T} M f+\gamma(f-y)^{T} D(f-y)\right)$

In the above equation (1) the variable ' $\mathrm{f}$ ' is related to the class labels of all the data , ' $y$ ' represents the class labels of the labeled data and ' $D$ ' is the diagonal matrix whose elements are ' 1 ' for labeled data and ' 0 ' for non labeled data, lastly the parameter $\gamma$ is a trade off parameter. The main task is to construct the matrix 'M'.

To achieve this, the splines developed in [9] are used replace the linear kernel function and models an interactive segmentation through spline based regressive process. The spline here is a combination of polynomial and Green's function that are used to interpolate the scattered data in geometrical coordinates. As per the work done in [1], it was observed that the matrix ' $M$ ' is Laplacian framework and can be obtained by summing the losses estimated from the neighborhoods.

In this paper, a two class labeling is considered, where the class label $f_{i}$, of $x_{i}$ belongs to the two valued label $L=\{+1,-1\}$. For each point of data $x_{i} \in X$, its " $\mathrm{k}$ " number of neighbors are selected based on the Euclidean distance then those closest data points can be represented as $N_{i}=\left\{x_{i j}\right\}_{j=1}^{k}$, where the ' $\mathrm{j}$ ' stands for the index and the next objective is to model a function that can route the data points to its labels, that can be represented as $f_{i j}=g\left(x_{i j}\right)$ Where $j=1,2, \ldots \mathrm{k}$.

For the above expression, the regression task can be modeled with a data fitting and smoothing function

$J(g)=\sum_{j=1}^{k}\left(f_{i j}-g i\left(x_{i j}\right)\right)^{2}+\lambda S(f)$ 
In the above equation (2) $\mathrm{S}(\mathrm{f})$ is a smoothing penalty on the function ' $\mathrm{f}$ ' and $\lambda$ is regularization parameter. Now, the problem of segmenting the foreground and background can be formulated as described in next section

\section{Proposed Approach}

Let us consider an Image ' $I$ ' which is labeled with user interaction into foreground and back ground regions. A class label of +1 is assigned fro foreground and class label of -1 is assigned for background, using the regressive approach stated in section 2 this problem can me mathematically formulated as

$\left.J(f)=\sum_{i=1}^{n f}\left(1-f\left(X_{i}^{F}\right)\right)^{2}+\sum_{i=1}^{n b}\left(1-f\left(X_{i}^{B}\right)\right)^{2}\right)$

Here in the above equation (3) "nf" are the number of foreground points and " $\mathrm{nb}$ " is the background points. Then the equation (2) can re-represent as equation (3).

The term $\mathrm{f}(\mathrm{x})$ termed as spline function can be given as in equation (4)

$f(x)=\alpha_{0}+\sum_{i=1}^{n} \alpha_{i} x_{i}+\sum_{j=1}^{n f} \varphi_{j}^{F}(x)+\sum_{j=1}^{n b} \varphi_{j}^{B}(x)$

In the above equation (4) the Green's function $\varphi_{j}^{F}(x)=$ $\left|x-x_{j}^{F}\right|^{2} \log \left(\left|x-x^{F}\right|\right)$ and similarly for $\varphi_{j}^{B}(x)$. To construct the matrix "M", all theses points are subjected for clustering. In this paper three clustering approaches like K-means [10], Spatial fuzzy C- means [11] and Sparsified K-means [12] are applied. The partitioned segments are indexed based on the thresholding,

$l_{i}= \begin{cases}\text { foreground } & \text { if } f(x) \geq 0 \\ \text { background } & \text { if } f(x)<0\end{cases}$

Thus obtained labeled points are combined to form a foreground and background regions.

\section{Experimental Results}

To evaluate the performance of the proposed approach, the algorithm is developed using matlab 2016 a version tool on Intel i5 processor and 8GB RAM system. The approach is compared against the graph cut [16]. The experiments are conducted with two standard benchmark datasets, Berkeley segmentation dataset available at [14] and Weizmann Segmentation Evaluation dataset available at [15] [20]
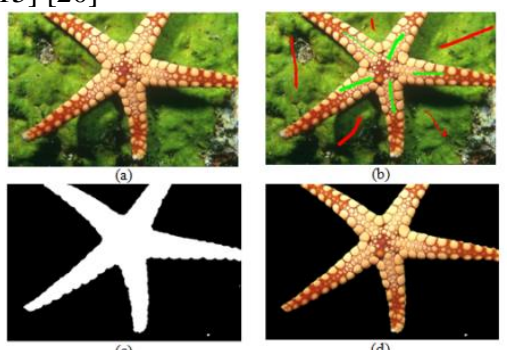

Figure 1: (a) Original Image (b) Labeled Image (c) Segmented Output (binary Mask) (d) Extracted Segmented Region
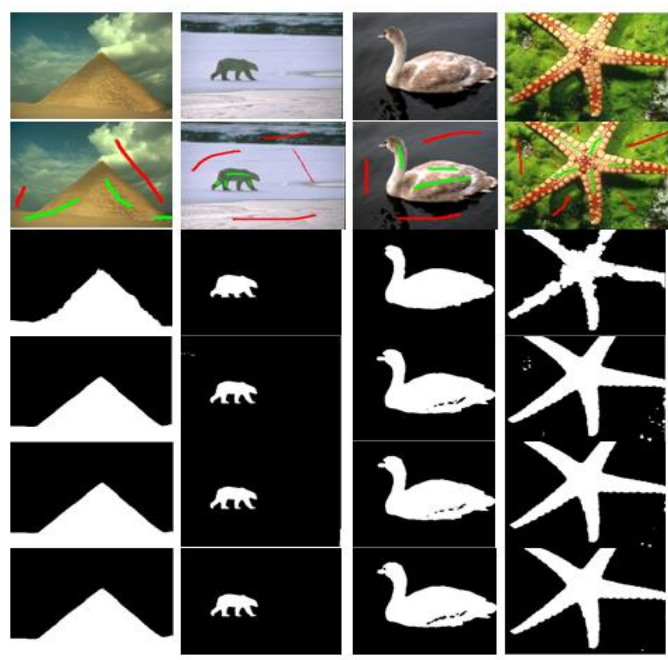

Figure 2: (a) First Row represents original images (b) Second Row represents Labeled Images (c) Segmented outputs of Grow cut [16], (d) Segmented outputs of Spline K-means [17] (e) Segmented Output of Spline SFCM [17] (f) Segmented Output of Spline regression based Sparse K-Means (proposed)

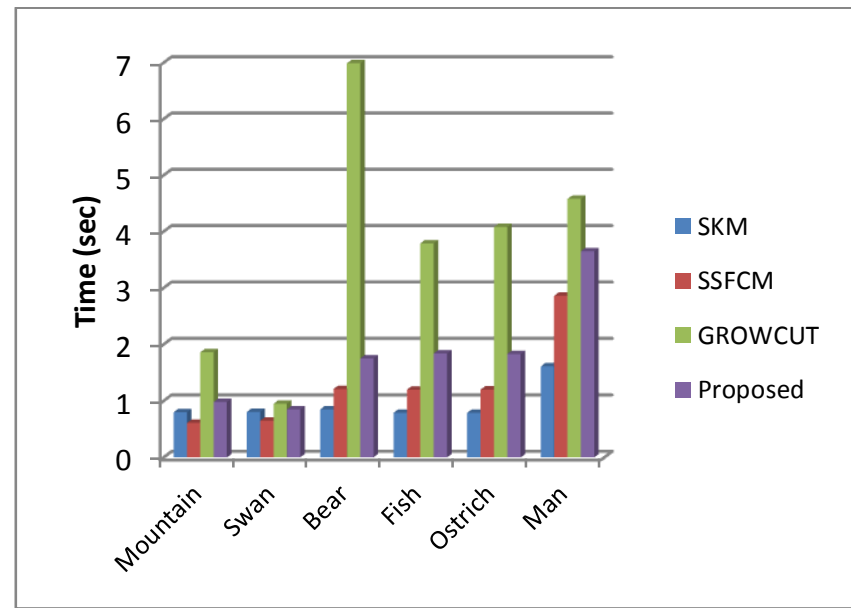

Figure 3: Time complexity analysis for different approaches

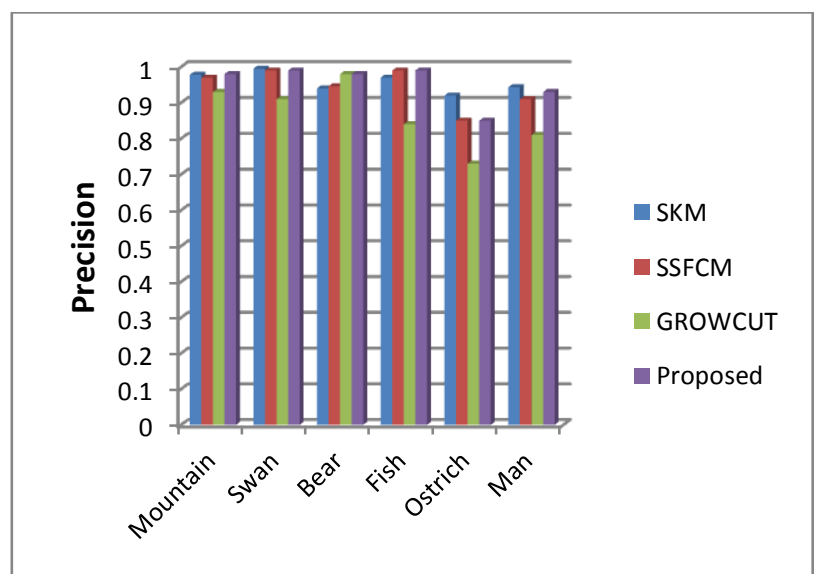

Figure 4: Precision analysis of different approaches

To evaluate the performance of the approaches 60 images from two datasets were considered few of them are displayed in figure 1 $\& 2$. For the evaluation time complexity, precision and recall [18] metrics were calculated. The performance of these metrics are depicted in figures $3,4 \& 5$, from these figure it can be observed that though the proposed approach is consuming 20 30\% more time, it is attaining more accurate results than earlier mentioned methods. [19] 


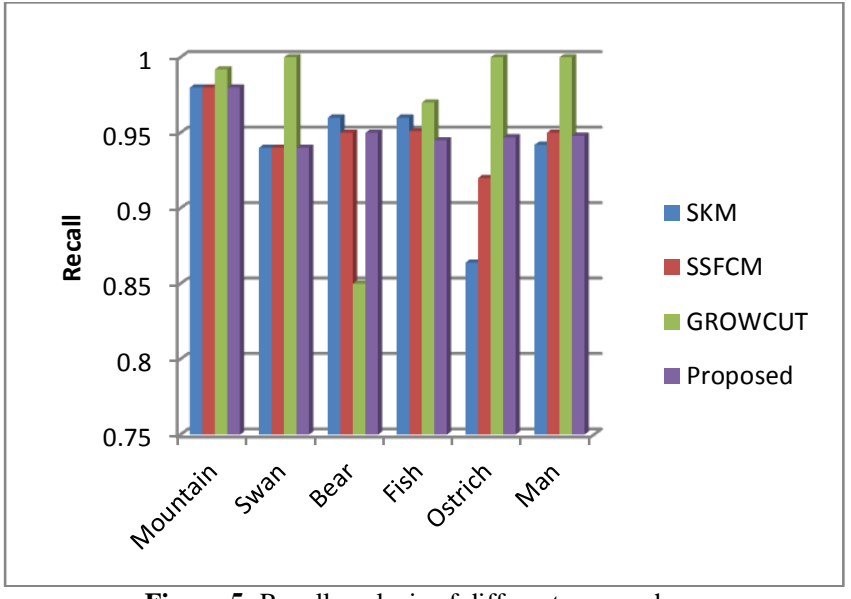

\section{Conclusion}

The paper presents a new sparse K-means based regression approach for natural image segmentation. Though the Sparse Kmeans is faster than K-means, due to the regression process it back drops in processing time however the segmentation results are attaining higher precision values than the earlier $\mathrm{K}$ means, SFCM and grow cut based approaches. This is can be treated as the achievement in the paper. The figures clearly show the fine extracted objects from the natural images which makes this algorithm to be more Venerable for object tracking and classification applications. This work can be put forwarded with the integration of deep neural networks.

\section{References}

[1] Xiang S, Nie F \& Zhang C, "Semi-Supervised Classification via Local Spline Regression", IEEE Transactions on Pattern Analysis and Machine Intelligence, Vol.32, No.11, (2010), pp.2039-2053.

[2] Xin J, Renjie Z \& Shengdong N, "Image Segmentation Based on Level Set Method", Physics Procedia, Vol.33, (2012).

[3] Kass M, Witkin A \& Terzopoulos D, "Snakes: Active contou models", International Journal of Computer Vision, Vol.2, (1988), pp.321-331.

[4] Cousty J, Bertrand G, Najman L \& Couprie M, "Watershed Cuts: Minimum Spanning Forests and the Drop of Water Principle", IEEE Transactions on Pattern Analysis and Machine Intelligence, Vol.31, No.8, (2009), pp.1362-1374

[5] Grady, L, "Random Walks for Image Segmentation", IEEE Transactions on Pattern Analysis and Machine Intelligence, Vol.28, No.11, (2006), pp.1768-1783.

[6] Boykov Y \& Funka Lea G, "Graph cuts and efficient image segmentation", International Journal of Computer Vision, Vol.69, No.2, (2006), pp.109-131.

[7] Zhou D, Bousquet O, Lal T, Weston J \& Scholkopf B, "Learning with local and global consistency", Adv. Neural Inf. Process. Syst. (2003), pp.321-328.

[8] Wang F \& Zhang C, "Label propagation through linear neighborhoods", IEEE Transactions on Knowledge and Data Engineering, Vol.20, No.1, (2008), pp.55-67.

[9] Ando RK \& Zhang T, "Two-view feature generation model for semi supervised learning”, International Conference on Machine Learning, (2007), pp. 25-32.

[10] A Tutorial on Clustering Algorithms Introduction, K-means, Fuzzy C-means, Hierarchical, Mixture of Gaussians https://webcache.googleusercontent.com

[11] Selvathi D \& Dhivya R, "Segmentation of tissues in MR images using Modified Spatial Fuzzy C Means algorithm”, International Conference on Signal Processing, Image Processing \& Pattern Recognition, (2013), pp. 136-140.

[12] Pourkamali-Anaraki, F \& Becker, S. Preconditioned data sparsification for big data with applications to PCA and Kmeans. IEEE Transactions on Information Theory, Vol.63, No.5, (2017), pp.2954-2974.
[13] Cai W, Chen S \& Zhang D, "Fast and robust fuzzy c-means clustering algorithms incorporating local information for image segmentation", Pattern Recognit., Vol.40, No.3, (2007), pp.825-838

[14] https://www2.eecs.berkeley.edu/Research/Projects/CS/vision/bsds/

[15] https://docs.opencv.org/3.0beta/modules/datasets/doc/datasets/is_weizmann.html

[16] Vezhnevets V \& Konouchine V, "GrowCut: Interactive multi-label ND image segmentation by cellular automata", Proc. of Graphicon., Vol.1, (2005), pp.150-156.

[17] David SRY \& Krishna RD, "Interactive Natural Image Segmentation With Regression Based Clustering Algorithm", International Journal of Latest Trends in Engineering and Technology Vol.8, No. 1, (2015), pp.494-497

[18] Yi X \& Eramian M, "LBP-Based Segmentation of Defocus Blur", IEEE Transactions on Image Processing, Vol.25, No.4, (2016), Pp.1626-1638.

[19] Villalobos Antúnez, JV (2017). Karl R. Popper, Heráclito y la invención del logos. Un contexto para la Filosofía de las Ciencias Sociales. Opción Vol. 33, Núm. 84. 5-11

[20] M Pallarès Piquer and O Chiva Bartoll (2017). La teoría de la educación desde la filosofía de Xavier Zubiri. Opción, Año 33, No. 82 (2017): $91-113$ 